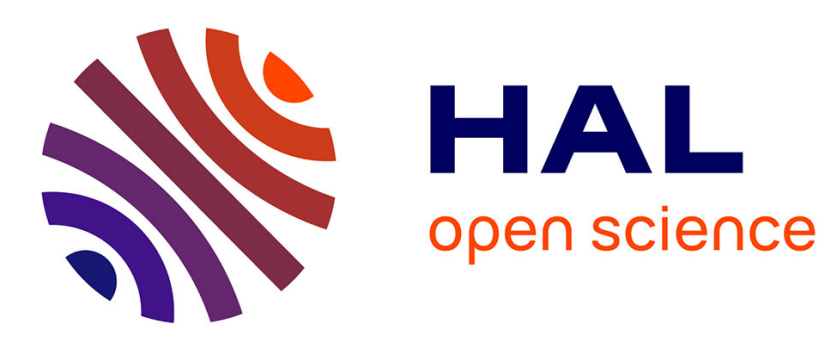

\title{
Constitutive Models and Numerical Methods for Frictional Contact
}

\author{
Michel Raous
}

\section{To cite this version:}

Michel Raous. Constitutive Models and Numerical Methods for Frictional Contact. Jean Lemaitre (Ed). Handbook of Materials Behaviour Models, Academic Press, pp 777-786 section 8.6, 2001, 0-12443343-X. hal-03178297

\section{HAL Id: hal-03178297 https://hal.science/hal-03178297}

Submitted on 24 Mar 2021

HAL is a multi-disciplinary open access archive for the deposit and dissemination of scientific research documents, whether they are published or not. The documents may come from teaching and research institutions in France or abroad, or from public or private research centers.
L'archive ouverte pluridisciplinaire HAL, est destinée au dépôt et à la diffusion de documents scientifiques de niveau recherche, publiés ou non, émanant des établissements d'enseignement et de recherche français ou étrangers, des laboratoires publics ou privés. 


\title{
Chapter 8 : Friction, Wear, Coating, Oxydation and Corrosion \\ Constitutive models and numerical methods for frictional contact
}

\author{
by Michel Raous \\ Directeur de Recherche au CNRS \\ Laboratoire de Mécanique et d'Acoustique \\ 31, chemin Joseph Aiguier \\ 13402 Marseille Cedex 20 - France
}

\section{Validity}

The following models are adapted to treat:

- unilateral contact, which means that penetration into the obstacle cannot occur and separation is free (the effective contact area depends on the loading),

- friction, which means that on the parts of the boundary where contact is established, a frictional force occurs.

The formulations and the algorithms are given under the assumptions that:

- contact occurs between a deformable body and a rigid obstacle (extension to contact between two deformable bodies is given as a remark),

- the deformations are small,

- the solid is elastic,

- the problem is quasistatic,

- adhesion is not involved,

- physico-chemical forces are not involved in the contact.

Extensions to finite deformations, non linear behaviour, dynamics problems and coupling to adhesion can be found in [1] and in references given in [1].

\section{Models and identification of the parameters}

\subsection{Unilateral contact}

Model CI : strict unilateral contact (Signorini conditions)

$$
\left.\begin{array}{ll}
u_{N} & \leq 0 \\
F_{N} & \leq 0 \\
u_{N} F_{N} & =0
\end{array}\right\}
$$

where $F$, the unknown contact force density, and $u$, the displacement defined on the part on the boundary where the contact may occur, are decomposed into normal and tangential components ( $n$ is the outward unit vector to the boundary) : $u=u_{N} n+u_{T}$, $F=F_{N} n+F_{T}$.

This satisfactorily describes unilateral contact with the two possible conditions : either no contact, when $u_{N}<0$ and $F_{N}=0$, or contact, when $u_{N}=0$ and $F_{N} \leq 0$. This is a non smooth problem, and the complementarity problem (??) implies that $F_{N}$ is a multivalued application of $u_{N}$, see the graph on Fig.??. No parameter is needed. 
Remark 1 : in the case where an initial gap between the solid and the obstacle has to be considered, $u_{N}$ has to be replaced by $\left(u_{n}-d\right)$ where the function d, defined on the boundary, is the initial distance between the solid and the obstacle. When the obstacle moves, the function $d$ is also used to define its movement.

Remark 2 : for the contact between two deformable bodies, in small deformations, a point to point relationship can be used under making a simple change of variables : if $\mathrm{A}$ (solid 1) and B (solid 2) are two facing points, let $u_{A}{ }^{*}=u_{A}$ and $u_{B}{ }^{*}=u_{A}-u_{B}$.

\section{Model CII : compliance}

This model allows a penetration which is controlled by a strong reacting force as follows :

$$
F_{N}=-C_{n}\left(u_{N}\right)_{+}^{m_{n}}
$$

where $\left(u_{N}\right)_{+}$is zero when $u_{N}<0$ (no contact), and $\left(u_{N}\right)_{+}=u_{N}$ when $u_{N} \geq 0$. This problem is much more regular, because $F_{N}$ is now a function of $u_{N}$. Its graph is given on Fig.??. This model is simpler for both computational and mathematical reasons. Nevertheless, some penetration, depending of the choice of parameters $C_{n}$ and $m_{n}$, occurs.

Identification of $C_{n}$ and $m_{n}$. A strict identification of $C_{n}$ and $m_{n}$ is not possible. The choice is often made for computational reasons. Mechanical estimates can be obtained by considering the penetration tolerance relatively to the current normal pressure. Considerations on the behaviour of surface asperities, analytically or through computations, can be helpful.

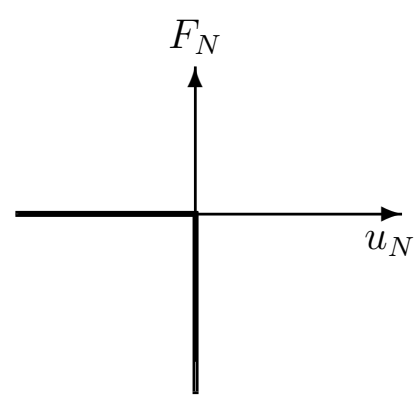

Figure 1: Graph of the Signorini law

Figure 2: Graph of the compliance law

\subsection{Friction}

The general form of a friction law can be written :

$$
\left.\begin{array}{l}
\left\|F_{T}\right\| \leq f \text { and } \\
\text { if }\left\|F_{T}\right\|<f \text { then } \dot{u}_{T}=0 \\
\text { if }\left\|F_{T}\right\|=f \text { then } \dot{u}_{T} \text { is colinear and opposite to } F_{T}
\end{array}\right\}
$$

where various choices for the sliding threshold $f$ can be made.

Model FI : Coulomb law, $f=\mu\left|F_{N}\right|$

This is the most classical friction law ( $\mu$ is the friction coefficient). 
Identification of the friction coefficient $\mu$. It is a very difficult matter because, firstly, this coefficient characterizes a pair of materials and, secondly it depends strongly on various conditions (surface machining, temperature, hygrometry, ...). Consequently, tables of friction coefficients are rarely given. As a qualitative indication, it can be said that the range of magnitude is about 0.1 to 0.3 for a metal/metal contact, larger than 1 for contact between rubber and another material and about 0.3 to 0.6 for a wood/wood contact.

Specific measurements can be undergone on a sample of the pair of materials by using a tribometer. When it is possible, it is recommended to identify this coefficient on the structure itself by adjusting this parameter, comparing a finite element computation to the experimental result for an elementary loading.

Model FII : Tresca law, $f=g$

The function $g$ is given and defined on the contact boundary. In this case, the sliding limit does not depend on the normal force. This model cannot be directly coupled with unilateral contact. It can be convenient when the normal force is known (polymer injection for example). It is useful in some iterative algorithms used to solve Coulomb problems.

Identification of the function $g$. When the normal pressure on the contact boundary is known, $\mathrm{g}$ is obtained by multiplying it by $\mu$, otherwise, some iterative procedure has to be used to determine a convenient value of the function $g$ on each contact point.

\section{Model FIII : variants}

- Coulomb-Orowan law, $f=\min \left(\mu\left|F_{N}\right|, k\right)$

The parameter $\mathrm{k}$ is usually the elastic limit of the material. It can be used when very high pressures are involved.

- Shaw law, $f=\alpha k$

The function $\alpha$ is defined by $\alpha=\Gamma_{R} / \Gamma_{C}$ where $\Gamma_{R}$ is the nominal (real) contact surface depending on the flattening of the asperities, and $\Gamma_{C}$ the whole supposed contact surface. This function depending on the normal compression has to be determined either analytically or numerically.

\section{Model FIV : models with a variable friction coefficient}

A dependence of the friction coefficient on either the sliding velocity (the most classical), the normal pressure, the temperature, or other quantities can be introduced. The Stribeck law (see "Friction in lubricated contacts" by J. Frêne and T. Cicone in this volume) is an example of such a dependence allowing to take into account the transition between dry friction and hydrodynamics regime.

Note : in spite of the attractiveness of this kind of model, one has to be aware of the loss of uniqueness (even for small friction coefficients) and the lack of existence results. This can lead to serious problems in so far as the reliability of the numerical results is concerned.

\section{Model FV : regularized Coulomb law.}

A regularized formulation of the Coulomb law is obtained when $F_{T}$ is given as a function of $\dot{u}_{T}$ (instead of the multivalued application), $F_{T}=-\mu \varphi_{\varepsilon}\left(\dot{u}_{T}\right)\left|F_{N}\right|$. For 2D problems, square root, hyperbolic tangent or piecewise polynomial functions are often used. In these cases, a parameter of regularization has to be chosen and the stick situation occurs only when $F_{T}=0$. 


\section{Model FVI : compliance model.}

As in the case of unilateral contact, a compliance law is introduced for the friction law :

$$
\left.\begin{array}{l}
\left\|F_{T}\right\| \leq C_{t}\left(u_{N}\right)_{+}^{m_{t}} \text { and } \\
\text { if }\left\|F_{T}\right\|<C_{t}\left(u_{N}\right)_{+}^{m_{t}} \text { then } \dot{u}_{T}=0 \\
\text { if }\left\|F_{T}\right\|=C_{t}\left(u_{N}\right)_{+}^{m_{t}} \text { then } \dot{u}_{T} \text { is colinear and opposite to } F_{T}
\end{array}\right\}
$$

where $C_{t}$ and $m_{t}$ are two parameters. The case where $C_{t}=C_{n}$ and $m_{t}=m_{n}$ corresponds to the classical Coulomb law associated with the compliance model for the unilateral contact. The same difficulties as for $C_{n}$ and $m_{n}$ occur for identifying $C_{t}$ and $m_{t}$.

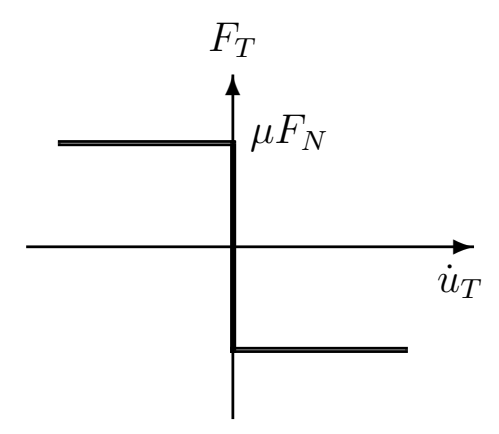

Figure 3: Graph of the Coulomb law (in 2D) Figure 4: Various friction laws (in 2D)

\section{Numerical methods}

\subsection{Formulations}

Both the displacements (unilateral conditions) and the velocities (friction law) are involved. When a displacement formulation is used, an incremental formulation has to be introduced. For each step, a "static" problem will be solved, involving extra terms computed at the previous step and characterizing the memory of the previous loading history. This section focuses on the resolution of this associated "static" problem.

The first three formulations concern models using the strict contact relationships (models CI, FI, FII, FIII, FIV), and the fourth formulation is related to models using the regularized contact relationships (models CII, FV, FVI).

\section{An implicit variational inequality}

Elimination of the contact forces leads to an implicit variational inequality. For example, a Signorini problem with Coulomb friction can be written as Problem $P_{1}$.

Problem $P_{1}$ : For each time $t_{k+1}$, find $u^{k+1} \in K$ such that :

$$
a\left(u^{k+1}, w-u^{k+1}\right)+j\left(u^{k+1}, w-u^{k}\right)-j\left(u^{k+1}, u^{k+1}-u^{k}\right) \geq L^{k+1}\left(w-u^{k+1}\right) \quad \forall w \in K
$$

where $\mathrm{a}(. .$.$) is a bilinear form associated to the elasticity mapping, \mathrm{L}($.$) is a linear form$ associated to the current loading, the non differentiable functional $j(v, w)$ (associated to 
the friction) is $j(u, v)=\int_{\Gamma_{C}} \mu\left|F_{N}(u)\right|\left\|v_{T}\right\| d s$ and the convex $\mathrm{K}$ characterizes the unilateral conditions $K=\left\{v \in U / v_{N} \leq 0\right.$ on $\left.\Gamma_{C}\right\}$ where $\Gamma_{C}$ is the part of the boundary where contact may occur.

\section{A complementarity problem}

An alternative is to write the problem as a linear complementarity problem, introducing two new variables by separating the tangential displacement into left and right sliding. It is then written, after FEM discretization and condensation of the problem to the contact variables (partial inversion of the linear parts), as problem $P_{2}$.

Problem $P_{2}$ : Find $F \in R^{p}, u \in R^{p}$ such that

$$
\left.\begin{array}{l}
M u=F^{*}+F \\
F_{i} \leq 0, u_{i} \leq 0 \quad i=1 \ldots p \\
F_{i} u_{i}=0 \quad i=1 \ldots p
\end{array}\right\}
$$

where $\mathrm{M}$ and $F^{*}$ are respectively a non symmetric matrix and a loading vector deduced from the FEM problem by condensation (and taking into account the change of variables associated to the choice of new variables), $F$ and $u$ are the contact forces and the contact displacements, and $\mathrm{p}$ is the number of contact degrees of freedom. This $2 \mathrm{D}$ formulation can be extended to 3D problems using a polygonalization of the Coulomb cone.

\section{Lagrange multipliers}

Another alternative is based, as for the previous formulation, in keeping the contact forces $F$ as variables (Lagrange multipliers). This time, the unilateral conditions are kept under the form (??) but the friction conditions are written under the Kuhn-Tucker conditions (analogous to plasticity). This is written as problem $P_{3}$ where $K^{*}$ is the condensed regular FEM matrix.

Problem $P_{3}:$ Find $F \in R^{p}, \Delta \xi \in R^{p}$ such that

$$
\begin{aligned}
& K^{*} u=F^{*}+F \\
& u_{N} \leq 0, F_{N} \leq 0, u_{N} F_{N}=0 \\
& f_{s} \leq 0, \Delta u_{T}=-\Delta \xi \partial f_{s} / \partial F_{T}, \Delta \xi \geq 0, \Delta \xi f_{s}=0 \\
& f_{s}=\left\|F_{T}\right\|-\mu\left|F_{N}\right|
\end{aligned}
$$

\section{A smooth non linear formulation}

When compliance, regularized models or penalization techniques are used, non linear variational equalities are obtained. Penalization can be considered as a numerical form of the compliance laws previously introduced. The problem is then written in the generic following form.

Problem $P_{4}$ : Find $u \in R^{p}$ such that :

$$
K^{*} u=F^{*}+\phi_{N}\left(u_{N}\right)+\phi_{T}\left(u_{T}\right)
$$

where $\phi_{N}\left(u_{N}\right)$ is given either by the compliance law (??) or by a penalization (for example $\left.\epsilon\left(u_{N}\right)_{+}\right)$, and $\phi_{T}\left(u_{T}\right)$ will be adjusted during the iterations in order to satisfy the 
Coulomb law.

\subsection{Algorithms and numerical parameters}

For problem $P_{1}$, a fixed point procedure can be introduced to determine the tangential frictional force $F_{T}$ : then, at each step $n+1$, we have to solve a Tresca problem with a given sliding threshold $g^{n}$ which can be itself set as the following minimization problem $P_{1 b i s}$, and solution of $P_{1}$ is obtained when the process $g^{n+1}=\mu\left|F_{N}\left(u^{n}\right)\right|$ has converged.

Problem $P_{1 b i s}$ : Find $u^{n}$ such that $J\left(u^{n}\right) \leq J(v) \forall v \in K$ with :

$$
J(v)=1 / 2 a(v, v)-L(v)+\int_{\Gamma_{C}} g^{n}\left\|v_{T}\right\| d s
$$

This minimization problem under constraints $(\forall v \in K)$ of a non differentiable functional can be solved by using various algorithms.

a - Overrelaxation method (SSOR) with projection (Problem $P_{1 b i s}$ )

An optimal parameter of relaxation has to be determined by a trial procedure. The method is very robust but can be costly when extension to non linear problems is considered.

b - A Gauss-Seidel method accelerated with an Aitken procedure (Problem $\left.P_{1 b i s}\right)$

No numerical parameter is needed. Same remark as for $(a)$ can be made.

c - Conjugate gradient method with projection and regularization (Problem $\left.P_{1 b i s}\right)$

A special procedure is used to preserve the conjugation relationship between the descent directions. Computations of the gradients need a regularization of the friction law which introduces numerical parameters (see model FV). Specific preconditionning has to be used. A regularization parameter is needed.

d - Lemke method (Problem $P_{2}$ )

The complementarity problem $P_{2}$ is solved by using a mathematical programming method, Lemke method for example. This is a direct mathematical programming method based on pivoting techniques analogous to Simplex methods. It is a very powerful method. No parameter is needed.

e - Augmented Lagrangian with radial return (Problem $P_{3}$ )

Problem $P_{3}$ is solved with augmented Lagrangian procedure which introduces a penalization by defining :

$$
\begin{array}{r}
F_{N}=\left(\lambda_{N}-\epsilon_{N} u_{N}\right) \\
\Delta F_{T}=\Delta \lambda_{T}-\epsilon_{T}\left(u_{T}+\Delta \xi \partial f_{s} / \partial F_{T}\right)
\end{array}
$$

A Newton Raphson algorithm is associated to a radial return procedure. It is analogous to a procedure used in plasticity (even for finite deformations). It is a very powerful method, the accuracy (and also the computational cost) depends on the choice of $\epsilon_{T}, \epsilon_{N}$, and on the number of augmentations performed. 


\section{f - Newton-Raphson method for the penalized problem (Problem $P_{4}$ )}

Problem $P_{4}$ is a classical non linear problem where the normal contact force is directly computed during the resolution and the frictional force is adjusted during the iterations in order to verify the Coulomb law. The choice of the penalization parameters (or of the parameters of the compliance law), is delicate because it has a strong influence on the accuracy of the solution (penetration can be large) and on the computational time. Usually, a progressive decrease of the parameters is conducted during the iterations. Accuracy of the solution has always to be checked.

\section{g - Iterative procedure on boundary conditions}

A simple procedure based on iterations on adjusting boundary conditions in order to verify the unilateral and the friction conditions is sometimes used : at each iterate, $u_{N}$ is set to zero when penetration occurs, $\dot{u}_{T}$ is first supposed to be zero and, when $\left\|F_{T}\right\|>\mu\left|F_{N}\right|$ the previous condition is released but a frictional force is applied, colinear and opposite to $\dot{u}_{T}$ and such that $\left\|F_{T}\right\|=\mu\left|F_{N}\right|$. Despite the lack of convergence results, this procedure can be efficient for simple linear problems. In other case, the procedure may not converge.

\section{References}

1 - M. Raous, Quasistatic Signorini problem with Coulomb friction and coupling to adhesion, chapter 3 in "New developments in contact problems", P. Wriggers-P.Panagiotopoulos (Eds), CISM Courses and Lectures, n 384, Springer Verlag, Wien-New York, 1999, pp. $101-178$ 